\title{
Thermo-mechanical behaviour of a Ni-Ti-Cu melt spun alloy
}

\author{
R. Santamarta, J. Pons and E. Cesari \\ Departament de Fisica, Universitat de les Illes Balears, Cra. de Valldemossa $\mathrm{km} \mathrm{7.5,}$ \\ 07071 Palma de Mallorca, Balearic /slands, Spain
}

\begin{abstract}
The martensitic transformation of a melt spun $\mathrm{Ti}_{50} \mathrm{Ni}_{25} \mathrm{Cu}_{25}$ alloy (cubic B2 $\rightleftarrows$ orthorhombic $\mathrm{B} 19$ ) has been investigated by means of thermo-mechanical cycling. The alloy was initially amorphous and it has been characterised after a controlled crystallisation process using differential scanning calorimetry, X-ray diffraction, thermal cycling tests under constant load, tensile tests and transmission electron microscopy. The recoverable elongation and the transformation temperatures have been studied as a function of the applied stress. However a new phenomena not yet completely understood has been observed during the cycling experiments under constant load. For the direct transformation, although the net shape change is an elongation, the strain-temperature $\varepsilon(T)$ curve has a region with a local contraction (opposite to the external load). The equivalent effect is also observed in the reverse transformation curve. The "training ability" (to achieve the two way shape memory effect) shown by the melt spun studied ribbons is lower than in other $\mathrm{Ni}-\mathrm{Ti}$ ribbons.
\end{abstract}

\section{INTRODUCTION}

The very good mechanical and functional properties of the near equiatomic Ni-Ti alloys are responsible of the extended use of the NiTi based family in comparison to other shape memory alloys (SMA). However, several features of these alloys are not suitable for particular applications and several ways to extend its use have been explored. Additions of a third element to the binary alloy have been widely studied in order to modify the transformation temperatures range and hysteresis, to improve some mechanical properties, etc. [1-4] and cover specific requirements of potential and present applications. Moreover, alternative production techniques have been applied to develop SMA with lower processing cost.

The addition of copper to binary $\mathrm{Ni}$-Ti leads to a transformation with lower hysteresis and decreases the effect of cycling processes in the transformation [5-6]. In addition to this, a significant lost of sensitivity of the martensitic transformation temperatures with the composition and the cooling rate has been found in these ternary alloys; copper also prevents $\mathrm{Ti}_{3} \mathrm{Ni}_{4}$ precipitation [5]. The martensitic phase of the ternary Ni-Ti-Cu alloy depends on the amount of copper substituting nickel. If this amount is not much higher than 5 at \% the martensite structure is monoclinic B19', like in the near equiatomic binary alloy. If copper is present in a larger amount than 10 at $\%$, the low temperature phase is orthorhombic $\mathrm{B} 19$. Around 10 at \% it is possible to detect both kinds of martensites in the sequence B2 $\rightarrow \mathrm{B} 19 \rightarrow \mathrm{B} 19^{\prime}$ $[7,8]$.

Melt-spinning is a new non-conventional technique which makes cheaper and easier the manufacture of thin ribbons. The production of almost ready-to-use ribbons by this way reduces the grain size, therefore decreases the transformation temperatures, compared to the bulk material, and the mechanical properties can be improved $[9,10]$. The 25 at \% copper containing alloy, which is usually amorphous in as-spun state, has been recently studied by several authors in order to check the crystallisation process and its influence on the microstructure and martensitic transformation [11-12]. The aim of this work is to 
analyse the thermo-mechanical behaviour in a $\mathrm{Ni}_{25} \mathrm{Ti}_{50} \mathrm{Cu}_{25}$ melt-spun ribbon after a crystallisation process.

\section{EXPERIMENTAL PROCEDURE}

A $\mathrm{Ni}_{25} \mathrm{Ti}_{50} \mathrm{Cu}_{25}$ alloy was made by arc-melting in a helium atmosphere. Subsequently, thin ribbons were produced by planar flow casting under helium atmosphere. The material was ejected out at $1625 \mathrm{~K}$, with a pressure of $200 \mathrm{hPa}$ onto a $23 \mathrm{~m} / \mathrm{s}$ rotating copper wheel and the resulting ribbons were of good macroscopic quality, $35 \mu \mathrm{m}$ thick and $21 \mathrm{~mm}$ wide. The as-spun alloy was almost completely amorphous and a previous crystallisation, which has been performed during $3.5 \mathrm{~min}$. at $885 \mathrm{~K}$, has been carried out before our experiments.

Thermo-mechanical cycling under constant load (up to a maximum of $70 \mathrm{MPa}$ ) has been carried out in an own designed device. The crystallised specimens, around $1 \mathrm{~cm}$ in width and $3.5 \mathrm{~cm}$ in length, were stressed in a liquid cooling-heating bath and the sample length variation was measured with a Linear Variable Differential Transducer (LVDT) sensor. Specimens for tensile stress-strain tests were of similar width and around $4-5 \mathrm{~cm}$ in length and the experiments have been performed in a Zwick Z100 testing machine with a digital extensometer and a controlled temperature chamber. The microstructure has been observed with a Hitachi H600 transmission electron microscopy (TEM) operating at $100 \mathrm{kV}$. A X-Ray Difractometer (XRD) Siemens D5000 has been used in order to check the crystalline structure of the alloy and differential scanning calorimetry (DSC) to determine the transformation temperatures without applied load.

\section{RESULTS AND DISCUSION}

XRD experiments show that the as-spun ribbon is mainly amorphous although few peaks corresponding to the high temperature phase can be detected. However, no transformation has been observed with the DSC in the range from $235 \mathrm{~K}$ to $870 \mathrm{~K}$. After crystallisation, cooling and heating runs have been performed at the DSC and single step transformations have been observed although small satellites in both heating and cooling curves are placed before and after the main transformation peaks (Figure 1). The transformation temperatures of the ribbon, included in Table 1, have been calculated at the $10 \%$ and the $90 \%$ of the total enthalpy change after the crystallisation process and an ageing process at $770 \mathrm{~K}$. By

$\mathrm{XRD}$ and electron diffraction $\mathrm{a} \mathrm{B} 2 \rightleftharpoons \mathrm{B} 19$ transformation has been associated with the calorimetric peaks observed during cooling from austenite and heating from martensite. The calculated lattice parameters are $a=3.04 \AA$ for the $\mathrm{B} 2$ phase and $a=2.91 \AA, b=4.29 \AA$ and $c=4.49 \AA$ for the B19 (Figure 2), which are quite similar to the values obtained by other authors in bulk alloys with the same composition [6] and melt-spun ribbons with less copper content [13].
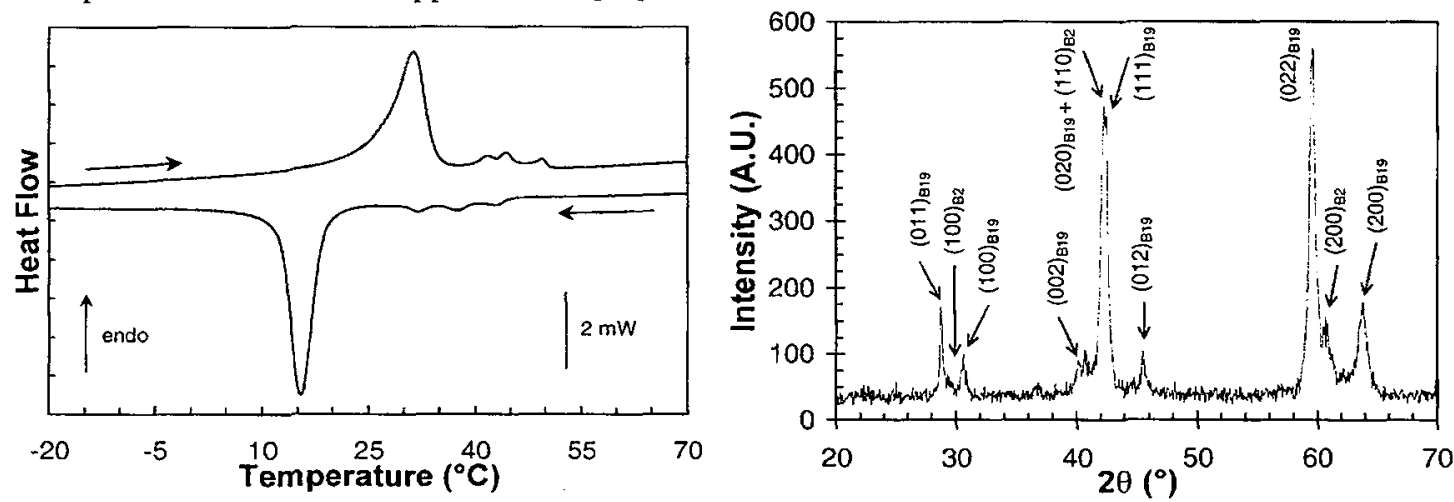

Figure 1. DSC curves upon cooling and heating of an ascrystallised $\mathrm{Ni}_{25} \mathrm{Ti}_{50} \mathrm{Cu}_{25}$ melt-spun alloy.

Figure 2. XRD pattern of a sample at room temperature ( $T \gtrsim A s$ ). Martensite and austenite peaks have been identified. 
Table1. Transformation temperatures, hysteresis and enthalpy change during the transformation of the $\mathrm{Ni}_{25} \mathrm{Ti}_{50} \mathrm{Cu}_{25}$ melt spun alloy after crystallisation with and without ageing treatment at $770 \mathrm{~K}$ in $\mathrm{Ar}$.

\begin{tabular}{|c|c|c|c|c|c|c|}
\hline Ageing time (h) & Ms (K) & Mf (K) & As (K) & Af (K) & $\Delta$ H (J/g) & Af-Mf (K) \\
\hline As-crystallised & 303 & 289 & 298 & 313 & 11.5 & 24 \\
\hline 1 & 299 & 291 & 295 & 315 & 10.4 & 24 \\
\hline 5 & 305 & 294 & 301 & 320 & 8.9 & 26 \\
\hline 23 & 309 & 295 & 311 & 323 & 7.6 & 28 \\
\hline
\end{tabular}

Strain as a function of temperature curves, $\varepsilon(T)$, obtained by thermal cycling under tensile constant load show the expected elongation on cooling and a shrinking on heating according to the applied load (Figures 3a,b). Nevertheless, in addition to this, an anomalous effect has been detected during the transformation consisting in a sharp contraction (on cooling) or elongation (on heating) in the opposite direction to the normal one which will be discussed below.
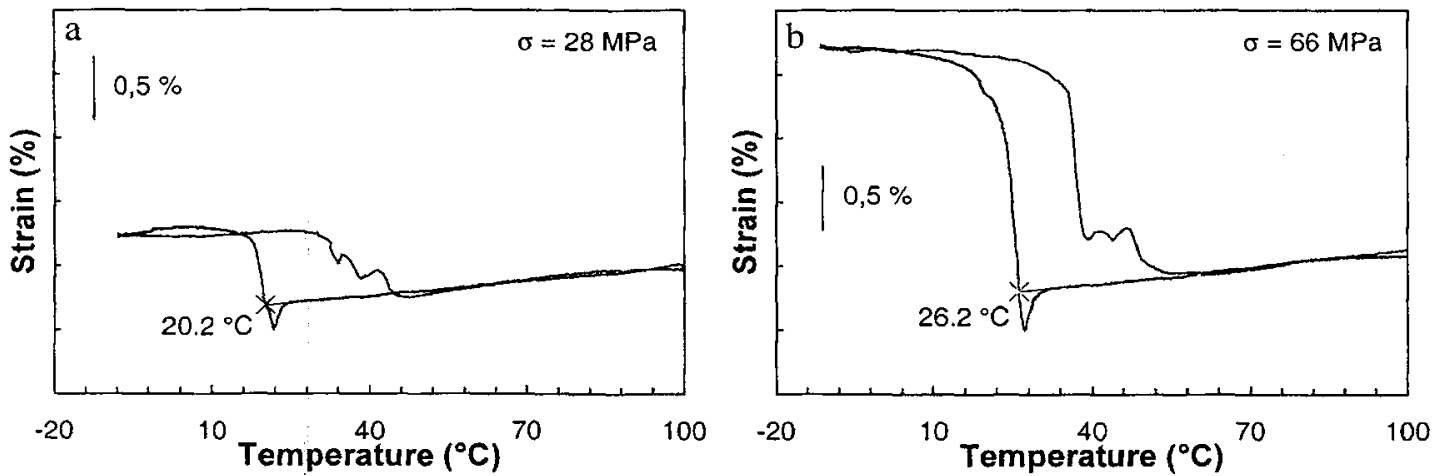

Figure 3. Thermo-mechanical cycles under $28 \mathrm{MPa}$ (a) and $66 \mathrm{MPa}$ (b) applied external load. Normal transformation and anomalous effect are clearly shown.

Several cycling series at increasing loads have been performed in order to check the reproducibility and evolution of the curves with cycling as well as the training capability. Cooling and heating curves differ slightly in the total strain associated to the direct and reverse transformation because the LVDT sensor begins to deviate from linearity below $260 \mathrm{~K}$. Also a non-completely horizontal baseline is obtained due to thermal dilatation. However, neither of these effects follows any significant influence in our temperatures or strain determination and they have not been corrected in the reported graphics.

The recoverable strain has been observed to increase continuously with the external load until the specimen fracture, which usually happened at around $60 \mathrm{MPa}$, the corresponding maximum recoverable strain being $\approx 2 \%$. Thus, the saturation of the shape memory effect could not be reached. The net strain estimated for the anomalous effect is about $0.2 \%$ in the specimen shown in Figure 3. However, it has to be pointed out that this value has a big dispersion for different samples.

No residual deformation has been observed during the performed cycles even in those which have been carried out with the maximum external force just before the fracture, so we can assure that the stress was not enough to achieve plastic deformation macroscopically detected. The existence of irregularities on both surfaces of melt-spun ribbons, inherent to the fabrication method, reduces the cross section at local places where stresses concentrate. These inhomogeneities, which can be of several $\mu \mathrm{m}$ in depth being the maximum thickness of $35 \mu \mathrm{m}$, have been detected observing the cross section of the melt-spun ribbon in an optical microscope. The stress concentration in these points can be enough to cause some non-measurable plastic deformation or even brittle fracture at these places. In fact, brittle fracture has been observed in tensile test of the $\mathrm{Ti}_{50} \mathrm{Ni}_{25} \mathrm{Cu}_{25}$ ribbons. Fracture strain is close to $1.5 \%$ for martensite (measured at room temperature) and the corresponding fracture stress is $150 \mathrm{MPa}$. Also the elastic 
modulus have been estimated, being $\mathrm{E}=35 \mathrm{GPa}$ for austenite (measured at $\mathrm{T} \approx 325 \mathrm{~K}$ ) and $\mathrm{E}=15 \mathrm{GPa}$ for martensite [14].

Several cycles at low stresses ( $\sigma \approx 4 \mathrm{MPa}$, to keep the ribbon extended) have been performed in highly-cycled samples in the stress range $30-55 \mathrm{MPa}$ in order to check the training capability of the ribbons. Within the experimental resolution of the thermo-mechanical cycling device no induced TWSME has been observed after about 70 cycles. However, a TWSME produced by bending has been reported in a melt-spun alloy with the same chemical composition and after several thermal treatments; the results exhibit the better effect after ageing at 773K [11]. Preliminary experiments with our alloy show no net elongation with thermo-mechanical cycling after 5 hours ageing at the same temperature, although there is transformation as DSC shows. Similar experiments without ageing have been carried out in an equiatomic NiTi melt-spun ribbon, obtaining a TWSME of $3 \%$ deformation after two series of thermo-mechanical cycling with increasing loads up to $60 \mathrm{MPa}$ [14]. An ageing treatment has been performed to the binary alloy at the same temperature obtaining also an important reduction of the elongation, while enthalpy change do not decrease considerably.

The dependence of the transformation temperatures, obtained as it is shown in Figures $3 a, b$, on the applied stress has been determined. The external stress dependence of the transformation temperature (or its inverse, do/dT), showing an approximated linear behaviour, has been calculated from the data of the direct transformation. The average slope of these points for all the checked specimens is $4.8 \pm 0.6 \mathrm{MPa} / \mathrm{K}$, which is quite different to the $\mathrm{do} / \mathrm{dT}$ estimated by other authors $(7.8 \mathrm{MPa} / \mathrm{K})$ in the $\mathrm{B} 2 \rightleftharpoons \mathrm{B} 19$ transformation for a $\mathrm{Ni}_{50} \mathrm{Ti}_{30} \mathrm{Cu}_{20}$ bulk alloy [8]. Some small differences have been found for low- and highly-cycled specimens, the latter having nearly bigger values than the former ones. However, this differences cannot be properly quantified as their values are lower than the uncertainties in $\mathrm{d} \sigma / \mathrm{dT}$.

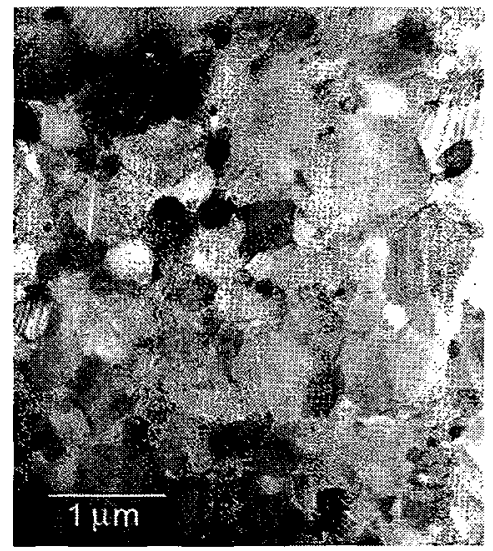

Figure 4.Low magnification micrograph showing the microstructure of the $\mathrm{Ti}_{50} \mathrm{Ni}_{25} \mathrm{Cu}_{25}$ ribbon after crystallisation.

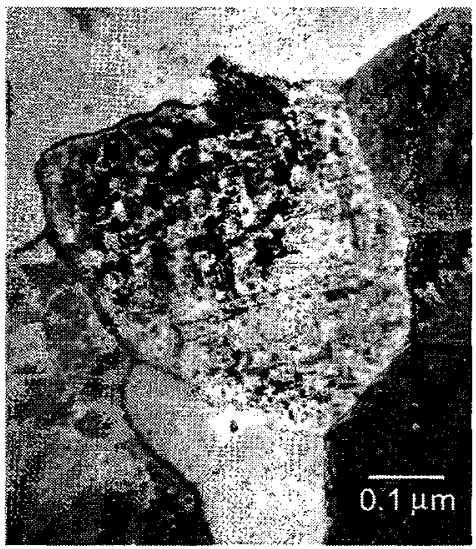

Figure 5. Example of a B2 grain with the particular distribution of linear defects.

Several samples from cycled and non-cycled specimens have been observed with the TEM and almost no differences have been noticed. The as-crystallised samples show a non-homogeneous distribution of the grain sizes, all of them being smaller than $1 \mu \mathrm{m}$ and many below $0.2 \mu \mathrm{m}$ in diameter (Figure 4). A very fine precipitate distribution is observed inside the grains, appearing as dark spots of few nm size. Another kind of defects in the form of linear traces has been also observed (Figure 5). Their appearance is similar to the plate precipitates lying on the $\{001\}_{\mathrm{B} 2}$ planes found after ageing a melt-spun $\mathrm{Ti}_{50} \mathrm{Ni}_{25} \mathrm{Cu}_{25}$ alloy [12] or a sputter-deposited $\mathrm{Ti}_{51.8} \mathrm{Ni}_{48.2}$ alloy [15]. The traces of these linear defects have been analysed in several pictures and only a part of them were consistent with the traces of $\{001\}_{B 2}$ planes. Therefore, it is not possible to rule out that some of them are dislocations. In fact, a slightly bigger 
presence of linear defects has been found in specimens with more than 100 thermo-mechanical cycles (many of them under stresses over $50 \mathrm{MPa}$ ) in relation to as-crystallised material. The additional linear defects observed in cycled specimens are most probably dislocations. In any case, their density is very low, which is consistent with the quite small differences found in the $\mathrm{d} \sigma / \mathrm{dT}$ values for cycled and noncycled specimens and the difficulties in the induction of the two-way shape memory effect.
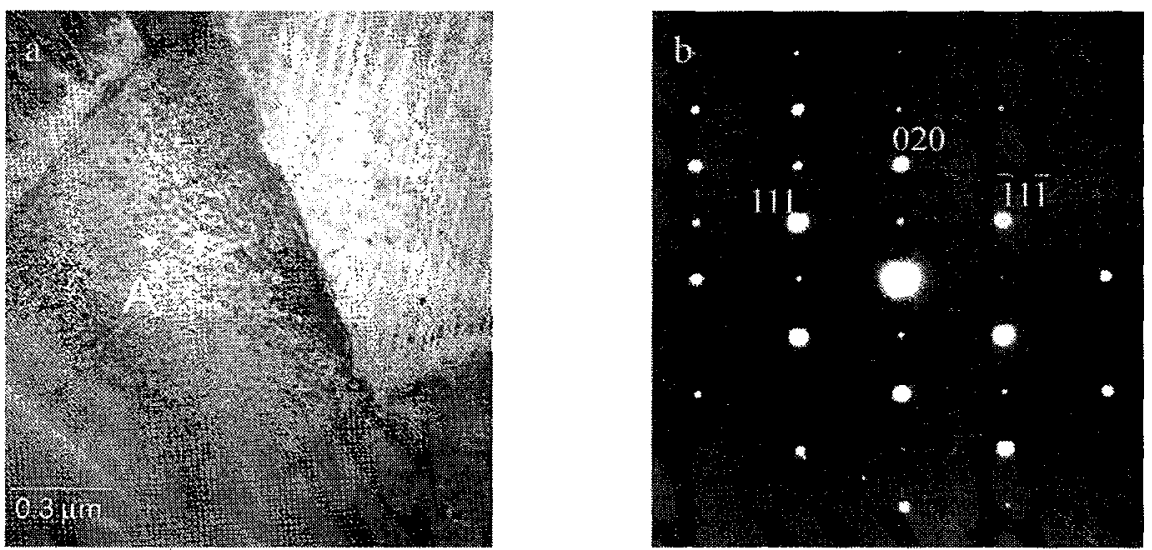

Figure 6. Bright field of a single martensite variant grain, labelled ' $A$ ' in the micrograph, surrounded by two-variant grains (a) and the corresponding selected-area electron diffraction pattern of grain A (b).

A particular situation, not usually present in shape memory alloys, has been observed both in ascrystallised and cycled samples. Some grains (mainly the biggest in size) exhibit the usual plate-type microstructure, characteristic of martensite, although the grains are filled mainly by two alternate variants, instead of forming complete groups of self-accommodating plates (with 4 variants). However, other grains show a uniform contrast throughout its extension, i.e. a contrast similar to the parent B2 phase, but the selected-area electron diffraction patterns correspond not to B2 structure but to a single B19 martensite variant. Thus, such observations confirm the existence of grains completely filled by a single martensite variant. Figures $6 \mathrm{a}, \mathrm{b}$ show an example of such grains and its diffraction pattern. The plate-type structure composed of self-accommodating variants is developed in order to minimise the shear strain and it is the common morphology for the temperature-induced transformation. The observation of one-variant grains suggests the existence of internal stresses particularly aligned in some of them, favouring the single variant microstructure. The role of the grain boundaries (with a much higher density in the ribbons than in bulk alloys) is believed to be the origin of such stresses.

The anomalous effect observed in all the crystalline specimens when they are thermo-mechanically cycled is not yet completely understood. This irregularity appears near the transformation temperatures obtained by means of the DSC curves, and it is completely reproducible. It has been found during either cooling or heating, always contrary to the expected response of a material under tensile load, with a similar hysteresis to the martensitic transformation one. It is necessary to note that is not an experimental artefact because it has not been observed in other melt-spun ribbons checked with the same device and parameters, like in a $\mathrm{Ni}_{50} \mathrm{Ti}_{50}$ ribbon whose transformation temperatures are contained in this range. As well, thermo-mechanical cycling has been performed in amorphous specimens of the copper content alloy presenting no anomalous effect, although the same specimens show it after the crystallisation process. The anomalous effect increases slightly with the applied load in the cooling branch, although not as much as the normal elongation as it can be seen in Figures 3.a and 3.b.

A hypothetical explanation for the appearance of the anomalous effect opposite to the external stress direction has been intended to find out. The strain around the grain boundaries and their associated 
stresses, which act in a high volume fraction in fine grained microstructures (like in the as-crystallised ribbon), are believed to be responsible for the appearance of a single martensite variant in many grains. These stresses can also promote the two-variant microstructure instead of full self-accommodating groups, as observed, as well as induce the transformation in some grains at higher temperatures than in the rest of the sample. It is not yet clearly understood why the observed particular martensite microstructure brings about a shortening of the length of the sample (against the external load) and its magnitude increases slightly, instead of decreasing, with rising loads.

\section{Acknowledgements}

Partial financial support from the DGESIC (project PB98-0127) is acknowledged. R.S. is grateful to UIB for a doctoral fellowship. Authors want to express their gratitude to J. Cifre, member of the S.C.T. (UIB), for his technical support in tensile tests.

\section{References}

1. J. Beyer and J.H. Mulder, Mat. Res. Soc. Symp. Proc. 360 (Materials Research Society, 1995) p. 443.

2. H. Hosoda, T. Fukul, K. Inoue, Y. Mishima and T. Suzuki, Res. Soc. Symp. Proc. 459 (Materials Research Society, 1997) p. 287.

3. V.A. Lobodyuk and M.M. Medyukh, Met. Phys. Adv. Tech. 16, 493 (1997).

4. M. Piao, S. Miyazaki, K. Otsuka and N. Nishida, Mat. Trans. JIM 33, 337 (1992).

5. T. Saburi, in Shape Memory Materials, edited by K. Otsuka and C.M. Wayman (Cambridge University Press, Cambridge, 1998), p. 49.

6. W.J. Moberly and K.N. Melton, in Engineering Aspects of Shape Memory Alloys, edited by T.W. Duerig, K.N. Melton, D. Stöckel and C.N. Wayman (Butterworth-Heinemann, Essex, 1990), p. 46.

7. T.H. Nam, T. Saburi, Y. Kawamura and K. Shimizu, Mat. Trans. JIM 31, 262 (1990).

8. T.H. Nam, T. Saburi and K. Shimizu, Mat. Trans. JIM 31, 959 (1990).

9. Y. Furuya, M. Matsumoto and T. Matsumoto, in Proceedings of ICOMAT-92, edited by C.M. Wayman and J.Perkins, (Monterey Institute of Advanced Studies Monterey, 1993), p.905.

10. S.D. Wang, X.Z. Wu, J.P. Zhang. H.Q. Su, J.L. Jin and B.T. Song, Trans. Mat. Res. Soc. Jpn. 18B, 1061 (1994).

11. A.V. Shelyakov, Y.A. Bykovsky, N.M. Matveeva and Y.K. Kovneristy, Journal de Physique IV C8-5, 713 (1995).

12. H. Rösner, A.V. Shelyakov, A.M. Glezer, K. Feit and P. Schloßmacher, Mat. Sci. and Eng. A273275, p. 733 (1999).

13. T.H. Nam, T. Saburi, Y. Nakata and K. Shimizu, Mat. Trans. JIM 31, p. 1050 (1990).

14. J. Pons, E. Cesari, R. Santamarta and V.A. Chernenko, Proceedings of EUROMAT 2000, Advances in Mechanical Behaviour, Plasticity and Damage, (in press).

15. T. Kikuchi, K. Ogawa, S. Kajiwara, T. Matsunaga and S. Miyazaki, in Proceedings of PTM'99 Conf., edited by M. Koiwa, K. Otsuka and T. Miyazaki (The Japan Institute of Metals, 1999), p. 1092. 